PROCEEDINGS OF THE

AMERICAN MATHEMATICAL SOCIETY

Volume 135, Number 7, July 2007, Pages 2283-2290

S 0002-9939(07)08759-X

Article electronically published on February 2, 2007

\title{
MAHARAM ALGEBRAS AND COHEN REALS
}

\author{
ILIJAS FARAH AND BOBAN VELIČKOVIĆ
}

(Communicated by Julia Knight)

\begin{abstract}
We show that the product of any two nonatomic Maharam algebras adds a Cohen real. As a corollary of this and a result of Shelah (1994) we obtain that the product of any two nonatomic ccc Souslin forcing notions adds a Cohen real.
\end{abstract}

\section{INTRODUCTION}

Given two forcing notions $\mathcal{P}$ and $\mathcal{Q}$ we write $\mathcal{P} \leq \mathcal{Q}$ iff forcing with $\mathcal{Q}$ introduces a $\mathcal{P}$-generic over $V$. This is equivalent to saying that there is $p \in \mathcal{P}$ and an embedding of the complete Boolean algebra $R O(\mathcal{P}\lceil p)$ into $R O(\mathcal{Q})$.

A forcing notion $\mathcal{Q}$ is basic if for any nontrivial poset $\mathcal{P} \leq \mathcal{Q}$ we have $\mathcal{Q} \leq \mathcal{P}$. Posets such as Cohen forcing, Random real forcing or Sacks forcing are basic in this sense. Let $\Sigma$ be a given class of posets. We say that $\Sigma_{0} \subseteq \Sigma$ is a basis for $\Sigma$ if for every $\mathcal{Q} \in \Sigma$ there is $\mathcal{P} \in \Sigma_{0}$ such that $\mathcal{P} \leq \mathcal{Q}$. It is not required that the members of $\Sigma_{0}$ be basic. The basis problem for the class of ccc posets asks if it is consistent that there be a small (i.e. finite) basis for this class of forcing notions. Another version of this problem is to identify a basis for the class of Souslin ccc posets. Recall that a poset $\mathcal{P}$ is Souslin if the domain of $\mathcal{P}$ is an analytic set of reals and both the ordering $\leq$ and the incompatibility relation $\perp$ of $\mathcal{P}$ are analytic subsets of $\mathbb{R}^{2}$. If we are willing to assume suitable large cardinal or determinacy assumptions, we can replace Souslin by some higher order definability. Clearly, both Cohen forcing $\mathcal{C}$ and random real forcing $\mathcal{R}$ should be members of any such basis. Prikry asked if it is consistent that $\{\mathcal{C}, \mathcal{R}\}$ form a basis for all ccc posets. This is equivalent to saying that every nontrivial ccc poset adds a Cohen or a random real. There is an obvious ZFC analog of this question for Souslin ccc posets.

Recall that a complete Boolean algebra is weakly distributive if for every double sequence $\left\{b_{n, k}\right\}_{n, k}$ of elements of $\mathcal{B}$ we have

$$
\bigwedge_{n} \bigvee_{k} b_{n, k}=\bigvee_{f: \mathbb{N} \rightarrow \mathbb{N}} \bigwedge_{n} \bigvee_{i<f(n)} b_{n, i} .
$$

Any measure algebra is weakly distributive. Clearly, a complete Boolean algebra $\mathcal{B}$ is weakly distributive iff every function in $\omega^{\omega}$ in a generic extension by $\mathcal{B}$ is

Received by the editors March 8, 2006.

2000 Mathematics Subject Classification. Primary 03Exx; Secondary 28Axx.

Key words and phrases. Exhaustive submeasure, Maharam algebra, Cohen real.

These results were obtained in December 2004 while the authors were visiting the E. Schrödinger Institute in Vienna. We would like to thank the Institute for providing hospitality and a stimulating environment. The first author was partially supported by NSERC.

(C)2007 American Mathematical Society 2283

Reverts to public domain 28 years from publication 
dominated by a function in the ground model. Therefore, we say that a partial ordering $\mathcal{P}$ is weakly distributive iff $R O(\mathcal{P})$ is weakly distributive. Concerning the question of Prikry, in [14] Shelah showed the following.

Theorem 1.1 (14]). If $\mathcal{P}$ is a nonatomic Souslin ccc forcing notion such that $\mathcal{P}\lceil p$ is not weakly distributive for any $p \in \mathcal{P}$, then $\mathcal{P}$ adds a Cohen real.

Since weakly distributive forcing notions cannot add a Cohen real, the remaining question is whether every weakly distributive ccc Souslin forcing notion adds a random real. Recall that a submeasure on Boolean algebra $\mathcal{B}$ is a function $\nu: \mathcal{B} \rightarrow$ $[0,1]$ such that

(1) $\nu(\mathbf{0})=0$.

(2) If $x \leq y$, then $\nu(x) \leq \nu(y)$.

(3) $\nu(x \vee y) \leq \nu(x)+\nu(y)$.

We say that $\nu$ is positive if $\nu(a)>0$, for every $a \in \mathcal{B} \backslash\{\mathbf{0}\}$. If $\mathcal{B}$ is complete the role of $\sigma$-additivity is played by the following continuity condition:

(4) $\nu\left(x_{n}\right) \rightarrow \nu\left(\inf _{n} x_{n}\right)$, whenever $\left\{x_{n}\right\}_{n}$ is a decreasing sequence.

A submeasure $\nu$ satisfying (4) is called continuous. If a complete Boolean algebra $\mathcal{B}$ carries a positive continuous submeasure, then we call it a Maharam algebra. The notion of a continuous submeasure was introduced by Maharam 12 who showed that every complete Boolean algebra carrying a positive continuous submeasure is weakly distributive and satisfies the ccc and who gave an algebraic characterization for a complete Boolean algebra to carry such a submeasure. The question of whether every Maharam algebra is a measure algebra was also raised in 12. This problem which is known to be equivalent to the Control Measure Problem (see, for instance, [7, [9, 13]) was the major open problem in this area until it was finally solved by Talagrand [16] in the fall of 2005.

Theorem 1.2 ([16]). There is a Maharam algebra which is not a measure algebra.

It is still not known if the algebra constructed by Talagrand adds a random real, i.e. if it has a complete subalgebra which is a measure algebra. On the other hand, Maharam algebras are the only ccc weakly distribute complete Boolean algebras which can be constructed in ZFC. Namely, it was shown by Balcar, Jech and Pazák [2] and Velickovic [19] that it is relatively consistent with ZFC (modulo the existence of a supercompact cardinal) that every ccc weakly distributive complete Boolean algebra is a Maharam algebra (for the statement and the relative consistency of the $P$-ideal dichotomy see [1] and [17]).

Theorem 1.3 ([2], 19]). For any $\kappa$ the P-ideal dichotomy for P-ideals on sets of size $\leq \kappa$ implies that every weakly distributive ccc complete Boolean algebra of size $\leq \kappa$ is a Maharam algebra.

As a corollary of the proof one obtains the following ZFC result which was also obtained independently by Farah and Zapletal [5].

Theorem 1.4 ([5]). If $\mathcal{P}$ is a weakly distributive Souslin ccc forcing notion, then $R O(\mathcal{P})$ is a Maharam algebra.

Thus, finding a basis for Souslin ccc forcing notions reduces to finding a basis for Maharam algebras. 
In this paper we take a different direction. Namely, it is well known that the product of any two nonatomic measure algebras adds a Cohen real (see for instance [3]). Motivated by this, the second author asked in [18 if the product of any two nonatomic Souslin ccc forcing notions adds a Cohen real. Here we give a positive answer to this question. In the process we establish that if $\varphi$ and $\psi$ are exhaustive submeasures on the algebra $\mathrm{CO}\left(2^{\mathbb{N}}\right)$ of clopen subsets of the Cantor space and at least one of them is pathological, then Fubini's theorem fails in the worst possible way for the product space. This extends previous results of Christensen [4] and Farah and Zapletal [5].

The paper is organized as follows. In $\S 1$ we present some facts about pathological submeasures and establish the strong failure of Fubini's theorem mentioned above. In $\S 2$ we show that the product of two nonatomic Maharam algebras adds a Cohen real and mention some corollaries. Our notation is mostly standard and can be found in $[8$ and $[6]$.

\section{Pathological submeasures and Fubini's theorem}

We say that a submeasure $\nu$ on a Boolean algebra $\mathcal{B}$ is exhaustive if for every sequence $\left(a_{n}\right)_{n}$ of pairwise disjoint elements of $\mathcal{B}$ we have $\lim _{n \rightarrow \infty} \nu\left(a_{n}\right)=0$. The reason this notion is important is the following. To any positive submeasure $\nu$ on a Boolean algebra $\mathcal{B}$ we can associate a metric $d_{\nu}$ on $\mathcal{B}$ by setting

$$
d_{\nu}(a, b)=\nu(a \Delta b),
$$

where $a \Delta b$ denotes the symmetric difference of $a$ and $b$. Let $\overline{\mathcal{B}}$ be the metric completion of $\mathcal{B}$ under this metric. The boolean operations extend to $\overline{\mathcal{B}}$ in the natural way. If $\nu$ is exhaustive, then $\overline{\mathcal{B}}$ is a complete Boolean algebra and $\nu$ can be extended to a continuous submeasure $\bar{\nu}$ on $\overline{\mathcal{B}}$, i.e., $\overline{\mathcal{B}}$ is a Maharam algebra. One concrete case of this construction is the following. Assume $\nu$ is an exhaustive submeasure on the algebra $\mathrm{CO}\left(2^{\mathbb{N}}\right)$ of clopen subsets of $2^{\mathbb{N}}$. Then one can mimic the usual construction of the Lebesgue measure to extend $\nu$ to a continuous submeasure $\bar{\nu}$ on the $\sigma$-algebra Borel of Borel subsets of $2^{\mathbb{N}}$. The submeasure $\bar{\nu}$ is not strictly positive, but if we let $\operatorname{Null}(\bar{\nu})$ denote the ideal of $\bar{\nu}$-null sets, then Borel / $\operatorname{Null}(\bar{\nu})$ is a Maharam algebra (see 7] for details).

Recall that a submeasure $\nu$ on a Boolean algebra is pathological if it does not dominate a positive nonzero finitely additive measure. The following is well known (see, for instance, [5, Lemma 2.5]).

Proposition 2.1. Let $\nu$ be a continuous submeasure on a complete Boolean algebra $\mathcal{B}$. Then there is $b \in \mathcal{B}$ such that $\nu \uparrow \mathcal{B}_{b}$ is pathological and $\nu \uparrow \mathcal{B}_{b} \mathrm{c}$ is equivalent to a measure, where $\mathcal{B}_{a}$ denotes the restriction of $\mathcal{B}$ to a.

We will use the following important result of Kalton and Roberts from [10] (see also [11] and [7]).

Theorem 2.2 ([10]). Let $\nu$ be a pathological submeasure on a Boolean algebra $\mathcal{B}$. Assume that $\nu\left(\mathbf{1}_{\mathcal{B}}\right)=1$ and let $\alpha<1 / 3$. Then for every integer $n$ there is a sequence $\left(a_{i}\right)_{i=1}^{n}$ of pairwise disjoint elements of $\mathcal{B}$ such that $\nu\left(a_{i}\right) \geq \alpha$, for all $i$.

Let $\mathrm{CO}\left(2^{\mathbb{N}}\right)$ denote the algebra of all clopen subsets of $2^{\mathbb{N}}$. For $A \subseteq\left(2^{\mathbb{N}}\right)^{2}$ let $A_{x}=\{y:(x, y) \in A\}$ and $A^{y}=\{x:(x, y) \in A\}$. Special cases of the following theorem appear in [4] and [5, Lemma 2.5]. 
Theorem 2.3. Let $\varphi$ and $\psi$ be exhaustive nonatomic submeasures on $\mathrm{CO}\left(2^{\mathbb{N}}\right)$. Assume that $\psi$ is pathological and let $\epsilon>0$. Then there is a clopen subset $W$ of $2^{\mathbb{N}} \times 2^{\mathbb{N}}$ such that

(1) $\psi\left(W_{x}^{\complement}\right) \leq \epsilon$, for every $x \in 2^{\mathbb{N}}$,

(2) $\varphi\left(W^{y}\right) \leq \epsilon$, for every $y \in 2^{\mathbb{N}}$.

Proof. First note that if $\nu$ is an exhaustive nonatomic submeasure on $\mathrm{CO}\left(2^{\mathbb{N}}\right)$, then for every $\delta>0$ there is an integer $n$ and a partition $\left(A_{i}\right)_{i=1}^{n}$ of $2^{\mathbb{N}}$ into disjoint clopen sets such that $\nu\left(A_{i}\right) \leq \epsilon$, for all $i$. We fix exhaustive submeasures $\varphi$ and $\psi$ as in the statement of the theorem.

Claim 2.4. For every $\epsilon>0$ there is a clopen set $U \subseteq 2^{\mathbb{N}} \times 2^{\mathbb{N}}$ such that

(1) $\psi\left(U_{x}\right) \geq \psi\left(2^{\mathbb{N}}\right) / 4$, for every $x \in 2^{\mathbb{N}}$,

(2) $\varphi\left(U^{y}\right) \leq \epsilon$, for every $y \in 2^{\mathbb{N}}$.

Proof. Fix a partition $\left(A_{i}\right)_{i=1}^{n}$ of $2^{\mathbb{N}}$ into disjoint clopen sets such that $\varphi\left(A_{i}\right) \leq \epsilon$, for all $i$. Now, since $\psi$ is pathological we can apply Theorem 2.2 to find a partition $\left(B_{i}\right)_{i=1}^{n}$ of $2^{\mathbb{N}}$ into clopen sets such that $\psi\left(B_{i}\right) \geq \psi\left(2^{\mathbb{N}}\right) / 4$, for all $i$. Let $U=$ $\bigcup_{i=1}^{n} A_{i} \times B_{i}$. Then $U$ is as desired.

Claim 2.5. Let $W$ be a clopen subset of $2^{\mathbb{N}} \times 2^{\mathbb{N}}$ and $\epsilon>0$. Then there is a clopen $U$ such that $U \cap W=\emptyset$ and

(1) $\psi\left(U_{x}\right) \geq \psi\left(\left(W_{x}\right)^{\complement}\right) / 4$, for every $x \in 2^{\mathbb{N}}$,

(2) $\varphi\left(U^{y}\right) \leq \epsilon$, for every $y \in 2^{\mathbb{N}}$.

Proof. Since $W$ is clopen we can fix an integer $n$ and a subset $S$ of $2^{n} \times 2^{n}$ such that $(x, y) \in W$ iff $\left(x\left\lceil n, y\lceil n) \in S\right.\right.$. For each $s \in 2^{<\mathbb{N}}$ let $[s]$ denote the set $\left\{x \in 2^{\mathbb{N}}: s \subseteq x\right\}$. For $s \in 2^{n}$ let $W_{s}$ denote $W_{x}$, for any (equivalently all) $x \in[s]$. Let $Z_{s}$ be the complement of $W_{s}$ and apply Claim 1 to get a clopen subset of $[s] \times Z_{s}$ such that

(1) $\psi\left(\left(U_{s}\right)_{x}\right) \geq \psi\left(Z_{s}\right) / 4$, for every $x \in[s]$,

(2) $\varphi\left(\left(U_{s}\right)^{y}\right) \leq \epsilon / 2^{n}$, for every $y \in Z_{s}$.

Let $U=\bigcup\left\{U_{s}: s \in 2^{n}\right\}$. Then $U$ is as desired.

To finish the proof of the theorem, using Claims 2.4 and 2.5 we construct a sequence $\left(U_{n}\right)_{n}$ of clopen subsets of $2^{\mathbb{N}} \times 2^{\mathbb{N}}$ such that, letting $W_{n}=\bigcup\left\{U_{i}: i<n\right\}$, for every $n$ we have

(1) $\psi\left(\left(U_{n}\right)_{x}\right) \geq \psi\left(\left(\left(W_{n}\right)_{x}\right)^{\complement}\right) / 4$, for every $x \in 2^{\mathbb{N}}$,

(2) $\varphi\left(\left(U_{n}\right)^{y}\right) \leq \epsilon / 2^{n+1}$, for every $y \in 2^{\mathbb{N}}$.

We claim that there is $n$ such that $\psi\left(\left(W_{n}\right)_{x}^{\complement}\right) \leq \epsilon$, for all $x \in 2^{\mathbb{N}}$. To see this assume otherwise and let $C_{n}$ be the set of all $x$ such that $\psi\left(\left(W_{n}\right)_{x}^{\complement}\right)>\epsilon$. Note that $C_{n}$ is clopen and by our assumption it is nonempty, for all $n$. Moreover $C_{n+1} \subseteq C_{n}$ for all $n$. Choose $x$ in $\bigcap_{n} C_{n}$. It follows that the sequence $\left(\left(U_{n}\right)_{x}\right)_{n}$ is pairwise disjoint and $\psi\left(\left(U_{n}\right)_{x}\right) \geq \epsilon / 4$. This contradicts the fact that $\psi$ is exhaustive.

We now have the following corollary, where Borel denotes the $\sigma$-algebra of all Borel subsets of $2^{\mathbb{N}}$.

Theorem 2.6. Assume $\varphi$ and $\psi$ are continuous nonatomic submeasures on Borel and $\psi$ is pathological. Then there is a Borel $A \subseteq\left(2^{\mathbb{N}}\right)^{2}$ such that $\psi\left(A_{x}\right)=0$ and $\varphi\left(\left(A^{y}\right)^{\complement}\right)=0$ for all $x, y$. 
Proof. Find $W(n)$ in $\mathrm{CO}\left(2^{\mathbb{N}}\right)$ such that $\psi\left(W(n)_{x}^{\complement}\right) \leq 2^{-n}$ and $\phi\left(W(n)^{y}\right) \leq 2^{-n}$ and let $A=\bigcap_{n} \bigcup_{m \geq n} W(m)$.

A continuous submeasure $\varphi$ is equivalent to a measure if there is a Borel probability measure $\mu$ such that $\operatorname{Null}(\varphi)=\operatorname{Null}(\mu)$. Two $\sigma$-ideals of Borel sets $\mathcal{I}, \mathcal{J}$ are orthogonal if there are $\mathcal{I}$ - and $\mathcal{J}$-positive $X, Y$ (respectively) and $A \subseteq X \times Y$, each of these sets Borel, such that $A_{x} \in \mathcal{J}$ and $Y \backslash A^{y} \in \mathcal{I}$ for all $x \in X$ and $y \in Y$.

Corollary 2.7. Assume $\varphi$ and $\psi$ are continuous nonatomic submeasures on Borel. Then exactly one of the following applies:

(1) Each one of $\varphi, \psi$ is equivalent to a measure.

(2) $\operatorname{Null}(\varphi)$ is orthogonal to $\operatorname{Null}(\psi)$.

Proof. Two possibilities are incompatible by Fubini's theorem. Assume (11) fails, so that one of $\varphi, \psi$ is not equivalent to a measure. We can assume it is $\psi$. Then there is a Borel set $A$ such that the restriction of $\psi$ to $A$ is pathological (see Proposition 2.1). Now we can apply Theorem 2.6.

\section{Adding Cohen Reals}

In this section we prove the main result of this paper.

Theorem 3.1. Let $\mathcal{B}$ and $\mathcal{C}$ be two nonatomic Maharam algebras. Then $\mathcal{B} \times \mathcal{C}$ adds a Cohen real.

Proof. First, we may assume without loss of generality that $\mathcal{B}$ and $\mathcal{C}$ are both countably generated. By the Loomis-Sikorski theorem we may also assume that there are continuous submeasures $\varphi$ and $\psi$ on Borel such that $\mathcal{B}=\operatorname{Borel} / \operatorname{Null}(\varphi)$ and $\mathcal{C}=\operatorname{Borel} / \operatorname{Null}(\psi)$. Since $\mathcal{B}$ and $\mathcal{C}$ are nonatomic, we may also assume that $\varphi$ and $\psi$ are positive on any nonempty clopen subset of $2^{\mathbb{N}}$. If both $\mathcal{B}$ and $\mathcal{C}$ are measure algebras, then $\mathcal{B} \times \mathcal{C}$ adds a Cohen real (e.g., [3, Theorem 3.2.11]). Therefore we may assume that one of $\mathcal{B}$ and $\mathcal{C}$ is not a measure algebra. By Proposition 2.1 for every continuous submeasure $\theta$ on Borel we can find a Borel set $A$ such that the restriction of $\theta$ to the algebra of Borel subsets of $A$ is equivalent to a measure, while the restriction of $\theta$ to the algebra of Borel subsets of $2^{\mathbb{N}} \backslash A$ is pathological. This means that without loss of generality we may assume that one of $\varphi$ or $\psi$ is a pathological submeasure. For concreteness, let us say that $\psi$ is pathological. Our plan is to use Theorem 2.3. For $\epsilon>0$, let us say that a clopen subset $U$ of $2^{\mathbb{N}} \times 2^{\mathbb{N}}$ is $\epsilon$-good if

(1) $\psi\left(U_{x}^{\complement}\right) \leq \epsilon$, for every $x \in 2^{\mathbb{N}}$,

(2) $\varphi\left(U^{y}\right) \leq \epsilon$, for every $y \in 2^{\mathbb{N}}$.

Thus, by Theorem 2.3 for every $\epsilon>0$ there is a clopen set $U$ which is $\epsilon$-good. Suppose now $U$ is $\epsilon$-good, for some $\epsilon>0$. Note that for every Borel sets $B$ and $C$ such that $\varphi(B)>\epsilon$ and $\psi(C)>\epsilon$ we have that $B \times C$ intersects both $U$ and $U^{\complement}$. Given $\rho, \epsilon$ and $\delta$ such that $0<\delta<\rho$, let us say that $U$ is $(\rho, \delta)$-good if for all Borel sets $B$ and $C$ with $\varphi(B)>\rho$ and $\psi(C)>\rho$ and $i \in\{0,1\}$ there are Borel sets $B^{i} \subseteq B$ and $C^{i} \subseteq C$ such that $\varphi\left(B^{i}\right)>\delta, \psi\left(C^{i}\right)>\delta$ and such that $B^{i} \times C^{i} \subseteq U^{i}$, where $U^{0}=U$ and $U^{1}=U^{\complement}$.

Claim 3.2. Suppose $U$ is clopen and $\epsilon$-good. Then for every $\rho>\epsilon$ there is $\delta>0$ such that $U$ is $(\rho, \delta)$-good. 
Proof. Since $U$ is clopen there is $n$ such that $U$ depends on the first $n$ coordinates, i.e., there is a subset $S$ of $2^{n} \times 2^{n}$ such that $(x, y) \in U$ iff $(x\lceil n, y\lceil n) \in S$. Let $\delta=\frac{\rho-\epsilon}{2^{n}}$. We will show that $U$ is $(\rho, \delta)$-good. Suppose $B$ and $C$ are Borel sets such that $\varphi(B)>\rho$ and $\psi(C)>\rho$. Let $T$ be the set of all $u \in 2^{n}$ such that $\varphi(B \cap[u])>\frac{\rho-\epsilon}{2^{n}}$. Let $B^{\prime}=\{x \in B: x\lceil n \in T\}$. Similarly let $R$ be the set of all $v \in 2^{n}$ such that $\psi(C \cap[v])>\frac{\rho-\epsilon}{2^{n}}$ and let $C^{\prime}=\{y \in C: y\lceil n \in R\}$. Then $\varphi\left(B^{\prime}\right)>\epsilon$ and $\psi\left(C^{\prime}\right)>\epsilon$. Since $U$ is $\epsilon$-good we have $B^{\prime} \times C^{\prime} \cap U \neq \emptyset$. Fix $(x, y) \in B^{\prime} \times C^{\prime} \cap U$. Let $u=x\lceil n$ and $v=y\lceil n$. It follows that $(u, v) \in S$, i.e. $[u] \times[v] \subseteq U$. Set $B^{0}=B \cap[u]$ and $C^{0}=C \cap[v]$. Then $B^{0}$ and $C^{0}$ are as desired. The construction of $B^{1}$ and $C^{1}$ is symmetric and is done in the same way.

We now construct a $\mathcal{B} \times \mathcal{C}$-name for a Cohen real. We are going to build a decreasing sequence of positive reals $\left(\epsilon_{n}\right)_{n}$ converging to 0 and a sequence $\left(U_{n}\right)_{n}$ of clopen subsets of $2^{\mathbb{N}} \times 2^{\mathbb{N}}$ such that $U_{n}$ is $\left(2 \epsilon_{n}, 2 \epsilon_{n+1}\right)$-good. To start let $\epsilon_{0}=1 / 2$ and let $U_{0}$ be any clopen subset of $2^{\mathbb{N}} \times 2^{\mathbb{N}}$ which is $1 / 2$-good. Given $\epsilon_{n}$ and $U_{n}$ which is $\epsilon_{n}$-good we first use Claim 3.2 to find $\epsilon_{n+1}$ such that $U_{n}$ is $\left(2 \epsilon_{n}, 2 \epsilon_{n+1}\right)$ good and then use Theorem 2.3 a to find a clopen subset $U_{n+1}$ of $2^{\mathbb{N}} \times 2^{\mathbb{N}}$ which is $\epsilon_{n+1}$-good. By decreasing $\epsilon_{n+1}$ if necessary we may assume that it is less than $1 / 2^{n+1}$. This completes the construction of the $\epsilon_{n}$ and $U_{n}$. Now, since each $U_{n}$ is clopen we can find an integer $m_{n}$ and a subset $S_{n}$ of $2^{m_{n}} \times 2^{m_{n}}$ such that $x \in U_{n}$ iff $x \uparrow m_{n} \in S_{n}$.

Suppose now $\dot{G} \times \dot{H}$ is the canonical name for the $\mathcal{B} \times \mathcal{C}$-generic filter. Let $\tau$ denote the name for the set of all $n$ such that there is $(u, v) \in S_{n}$ such that $[u] \times[v] \in \dot{G} \times \dot{H}$.

Claim 3.3. The maximal condition in $\mathcal{B} \times \mathcal{C}$ forces that $\tau$ is Cohen generic over the ground model.

Proof. Let $D$ be a dense set in the Cohen forcing $2^{<\mathbb{N}}$ and let $(B, C)$ be a condition in $\mathcal{B} \times \mathcal{C}$. Let $\rho=\min \{\varphi(B), \psi(C)\}$. Find $n$ such that $\rho>2 \epsilon_{n}$. Then find an integer $m$ and $t \in 2^{m}$ such that $\widehat{s t} \in D$, for every $s \in 2^{n}$. We build decreasing sequences $\left(B_{i}\right)_{i=0}^{m}$ and $\left(C_{i}\right)_{i=0}^{m}$ of conditions in $\mathcal{B}$ and $\mathcal{C}$, respectively. We will have $\varphi\left(B_{i}\right)>2 \epsilon_{n+i}$ and $\psi\left(C_{i}\right)>2 \epsilon_{n+i}$, for all $i$. To start let $B_{0}=B$ and $C_{0}=C$. Suppose $B_{i}$ and $C_{i}$ have been constructed. Since $U_{n+i}$ is $\left(2 \epsilon_{n+i}, 2 \epsilon_{n+i+1}\right)$ good if $t(i)=0$ we can choose $B_{i+1}$ and $C_{i+1}$ such that $\varphi\left(B_{i+1}\right)>2 \epsilon_{n+i+1}$ and $\psi\left(C_{i+1}\right)>2 \epsilon_{n+i+1}$ and such that $B_{i+1} \times C_{i+1} \cap U_{n+i}=\emptyset$. If $t(i)=1$ we can choose $B_{i+1}$ and $C_{i+1}$ such that $B_{i+1} \times C_{i+1} \subseteq U_{n+i}$. Finally let $B^{*}=B_{m}$ and $C^{*}=C_{m}$. It follows that $\left(B^{*}, C^{*}\right)$ forces that there is $s \in 2^{n}$ such that $\tau \uparrow(n+m)=\widehat{s} t$ and therefore $\tau \uparrow(n+m) \in D$. Since $B$ and $C$ were arbitrary it follows that $\tau$ is forced to be a Cohen real over the ground model.

This completes the proof of Claim 3.3 and Theorem 3.1 .

Using Theorems 1.1, 1.4 and 3.1 we now have the following immediate corollary.

Corollary 3.4. If $\mathcal{P}$ and $\mathcal{Q}$ are nonatomic Souslin ccc forcing notions, then $\mathcal{P} \times \mathcal{Q}$ adds a Cohen real.

Remark. The conclusion of Theorem 3.1 for exhaustive submeasures $\phi$ and $\psi$ on the reals is equivalent to the existence of a Borel-measurable function $f$ on $\mathbb{R}^{2}$ such that the image of every rectangle with positive Borel sides is nonmeager. As the referee pointed out, our proof shows that there is a continuous function on $\mathbb{R}^{2}$ such 
that the image of every rectangle with positive Borel sides includes a nonempty open set. This generalizes a well-known result of Steinhaus.

We now make some observations about forcing notions which add unbounded reals. First, recall that a subtree $T$ of $\mathbb{N}<\mathbb{N}$ is superperfect if for every $s \in T$

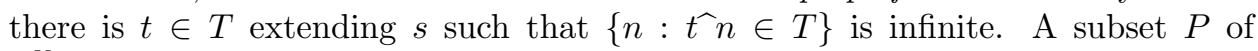
$\mathbb{N}^{\mathbb{N}}$ is superperfect if it is the set of all branches of a superperfect tree. In 20. Velickovic and Woodin define a continuous partial function $f: \mathbb{N}^{\mathbb{N}} \rightarrow 2^{\mathbb{N}}$ such that $f[P \times Q \times R]$ contains an interval for every triple of superperfect sets $P, Q, R$. Note that this implies that for every forcing notion $\mathcal{P}$ which adds an unbounded real, $\mathcal{P}^{3}$ adds a Cohen real. Namely, if $\tau$ is a $\mathcal{P}$-name for an unbounded real, let $\tau_{i}$, for $i=1,2,3$, be the copy of $\tau$ on the $i$-th coordinate. Then it is easy to see that $f\left(\tau_{1}, \tau_{2}, \tau_{3}\right)$ is forced to be Cohen generic over the ground model. This allows us to deduce the following corollary.

Corollary 3.5. It is relatively consistent with $Z F C$ that for every nonatomic ccc forcing notion $\mathcal{P}$ there is $p \in \mathcal{P}$ such that forcing with $\mathcal{P}^{3}$ below $(p, p, p)$ adds a Cohen real. Moreover, in the same model the product of any four nonatomic ccc forcing notions adds a Cohen real.

Proof. In [1] starting from any model of ZFC, Abraham and Todorcevic constructed a model of set theory in which the $P$-ideal dichotomy holds for $P$-ideals on sets of size at most $\mathfrak{c}$. We show that this model is as required. To see this let $\mathcal{P}$ be a nonatomic ccc forcing notion. By going to a regular subordering we may assume that $|\mathcal{P}| \leq \mathfrak{c}$. If $\mathcal{P}$ is weakly distributive, then by Theorem 1.3 the regular open algebra of $\mathcal{P}$ is a Maharam algebra and thus by Theorem $3.1 \mathcal{P}^{2}$ adds a Cohen real. If there is a condition $p \in \mathcal{P}$ which forces that an unbounded real is added, then by the above result from [20] forcing with $\mathcal{P}^{3}$ below $(p, p, p)$ adds a Cohen real.

To see the second statement, let $\mathcal{P}_{i}$ be nonatomic ccc forcing notions, for $i=$ $1, \ldots, 4$. Let $G=\prod_{i=1}^{4} G_{i}$ be a generic filter over the ground model. Then for each $i$ there is $p_{i} \in G_{i}$ such that forcing with $\mathcal{P}_{i}$ below $p_{i}$ is either weakly distributive or adds an unbounded real. If at least two of them are weakly distributive, then by Theorem $3.1 V[G]$ contains a Cohen real over the ground model. If for at least three different $i$ forcing with $\mathcal{P}_{i}$ below $p_{i}$ adds an unbounded real, then $V[G]$ contains a Cohen real over the ground model by the result from [20].

Remark. It has been observed by Goldstern (unpublished) that if a forcing notion $\mathcal{P}$ adds a dominating real, then $\mathcal{P}^{2}$ adds a Cohen real. This cannot be improved by replacing 'dominating real' by 'unbounded real'. Namely, let $\mathcal{M}$ denote Miller forcing with superperfect subtrees of $\mathbb{N}<\mathbb{N}$. It is known that $\mathcal{M}$ adds un unbounded real, but Spinas [15] showed that $\mathcal{M}^{2}$ does not add Cohen reals.

Finally we state some open problems related to the results of this paper.

Question 3.6. Does the complete Boolean algebra constructed by Talagrand [16] add a random real?

Question 3.7. Say that a forcing notion is absolutely ccc if $\mathcal{P}$ remains ccc in any generic extension of the universe with the same $\aleph_{1}$. If $\mathcal{P}$ is a nonatomic absolutely ccc forcing notion, does $\mathcal{P}^{2}$ add a Cohen real?

Question 3.8. Is it relatively consistent that every ccc forcing which adds an unbounded real adds a Cohen real? 


\section{REFERENCES}

1. U. Abraham and S. Todorčević, Partition properties of $\omega_{1}$ compatible with CH, Fund. Math. 152 (1997), no. 2, 165-181. MR1441232 (98b:03064)

2. B. Balcar, T. Jech, and T. Pazák, Complete ccc Boolean algebras, the order sequential topology, and a problem of von Neumann, Bulletin of the London Math. Soc. 37 (2005), no. 6, 885-898. MR2186722

3. T. Bartoszynski and H. Judah, Set theory: on the structure of the real line, A.K. Peters, 1995. MR 1350295 (96k:03002)

4. J.P.R. Christensen, Some results with relation to the control measure problem, Vector space measures and applications II (R.M. Aron and S. Dineen, eds.), Lecture Notes in Mathematics, vol. 645, Springer, 1978, pp. 27-34. MR0502425 (80e:28008)

5. I. Farah and J. Zapletal, Between Maharam's and von Neumann's problems, Math. Res. Lett. 11 (2004), no. 5-6, 673-684. MR.2106234(2005i:03059)

6. D.H. Fremlin, Measure theory, vol. 3, Torres-Fremlin, 2002.

7. Maharam algebras, preprint, University of Essex, available at http://www.essex.ac.uk/maths/staff/fremlin/preprints.htm, 2004.

8. Thomas Jech, Set theory, Springer Monographs in Mathematics, Springer-Verlag, Berlin, 2003, The third millennium edition, revised and expanded. MR.1940513 (2004g:03071)

9. Nigel Kalton, The Maharam problem, Séminaire d'initiation à l'analyse, Publ. Math. Univ. Pierre et Marie Curie, vol. 94, Univ. Paris VI, Paris, 1989, Exp. No. 18, 13 pp. MR.1107322 (92c:28005)

10. N.J. Kalton and J.W. Roberts, Uniformly exhaustive submeasures and nearly additive set functions, Transactions of the American Mathematical Society 278 (1983), 803-816. MR:0701524 (85f:28006)

11. A. Louveau, Progrès récents sur le problème de Maharam d'après N.J. Kalton et J.W. Roberts, Séminaire d'initiation à l'analyse, 28ème année (G. Choquet et al., ed.), no. 20, 1983/84, pp. 01-08.

12. Dorothy Maharam, An algebraic characterization of measure algebras, Ann. of Math. (2) 48 (1947), 154-167. MR0018718 (8:321b)

13. J.W. Roberts, Maharam's problem, Proceedings of the Orlitz memorial conference (P. Kranz and I. Labuda, eds.), 1991, unpublished.

14. S. Shelah, How special are Cohen and random forcings, i.e. Boolean algebras of the family of subsets of reals modulo meagre or null, Israel Journal of Mathematics 88 (1994), 159-174. MR.1303493 (96g:03090)

15. Otmar Spinas, Ramsey and freeness properties of Polish planes, Proc. London Math. Soc. (3) 82 (2001), no. 1, 31-63. MR.1794256 (2001j:03088)

16. Michel Talagrand, Maharam's problem, preprint, 31 pages, 2006.

17. S. Todorcevic, A dichotomy for P-ideals of countable sets, Fundamenta Mathematicae 166 (2000), 251-267. MR.1809418 (2001k:03111)

18. B. Veličković, The basis problem for ccc posets, Set Theory, DIMACS Series, vol. 58, American Mathematical Society, 2002, pp. 149-160. MR1903857 (2003e:03098)

19. _ CCC forcings and splitting reals, Israel Journal of Mathematics 147 (2005), 209-220. MR2166361 (2006d:03088)

20. Boban Veličković and W. Hugh Woodin, Complexity of reals in inner models of set theory, Ann. Pure Appl. Logic 92 (1998), no. 3, 283-295. MR.1640916 (99f:03067)

Department of Mathematics and Statistics, York University, 4700 Keele Street, North York, Ontario, Canada M3J 1P3

Current address: Matematički Institut, Kneza Mihaila 35, Beograd, Serbia and Montenegro

E-mail address: ifarah@mathstat.yorku.ca

URL: http://www.math.yorku.ca/ ifarah

Equipe de Logique Mathématique, UFR de Mathématiques (Case 7012), Université Denis-Diderot Paris 7, 2 place Jussieu, 75251 Paris Cedex 05, France

Current address: Matematički Institut, Kneza Mihaila 35, Beograd, Serbia and Montenegro

E-mail address: boban@logique.jussieu.fr

$U R L:$ http://www.logique.jussieu.fr/ boban 